\title{
Plasmonic metasurfaces with $42.3 \%$ transmission efficiency in the visible
}

\author{
Jihua Zhang ${ }^{1}$, Mohamed ElKabbash $\mathbb{1}^{1}$, Ran Wei', Subhash C. Singh ${ }^{1,2}$, Billy Lam ${ }^{1}$ and Chunlei Guo ${ }^{1,2}$
}

\begin{abstract}
Metasurfaces are two-dimensional nanoantenna arrays that can control the propagation of light at will. In particular, plasmonic metasurfaces feature ultrathin thicknesses, ease of fabrication, field confinement beyond the diffraction limit, superior nonlinear properties, and ultrafast performances. However, the technological relevance of plasmonic metasurfaces operating in the transmission mode at optical frequencies is questionable due to their limited efficiency. The state-of-the-art efficiency of geometric plasmonic metasurfaces at visible and near-infrared frequencies, for example, is $\leq 10 \%$. Here, we report a multipole-interference-based transmission-type geometric plasmonic metasurface with a polarization conversion efficiency that reaches $42.3 \%$ at $744 \mathrm{~nm}$, over $400 \%$ increase over the state of the art. The efficiency is augmented by breaking the scattering symmetry due to simultaneously approaching the generalized Kerker condition for two orthogonal polarizations. In addition, the design of the metasurface proposed in this study introduces an air gap between the antennas and the surrounding media that confines the field within the gap, which mitigates the crosstalk between meta-atoms and minimizes metallic absorption. The proposed metasurface is broadband, versatile, easy to fabricate, and highly tolerant to fabrication errors. We highlight the technological relevance of our plasmonic metasurface by demonstrating a transmission-type beam deflector and hologram with record efficiencies.
\end{abstract}

\section{Introduction}

Plasmonic metasurfaces (PMs) are two-dimensional arrays of metallic nanoantennas (meta-atoms) with subwavelength thicknesses and spacings and a spatially varying phase response ${ }^{1,2}$. PMs promise a paradigm shift in optics by replacing traditional bulky optical elements, e.g., beam deflectors ${ }^{3-6}$, lenses ${ }^{7-10}$, waveplates ${ }^{11,12}$, vortex generators $^{13-16}$, and holograms ${ }^{17-20}$ with ultrathin, lightweight, and easy-to-integrate two-dimensional optical interfaces. Furthermore, PMs have an ultrathin thickness on the order of tens of nanometers, are easy to fabricate, support a field confinement beyond the diffraction limit, and have potential to respond on the timescale of a few femtoseconds ${ }^{21}$; these properties are unparalleled in tra-

Correspondence: Chunlei Guo (guo@optics.rochester.edu)

${ }^{1}$ The Institute of Optics, University of Rochester, Rochester, NY 14627, USA

${ }^{2}$ Changchun Institute of Optics, Fine Mechanics, and Physics, Chinese

Academy of Sciences, 130033 Changchun, China ditional optical elements and cannot be easily extended to dielectric metasurfaces. Moreover, over the past two decades, the field of plasmonics has undergone significant advances and enabled a wide range of practical applications ${ }^{21-23}$. Applying the concept of PMs to these plasmonic devices and systems can further enhance their functionalities and performance, particularly in applications where plasmonics have unique advantages, such as superlens $^{24}$, quantum plasmonics ${ }^{25}$, nonlinear optics ${ }^{26}$, photovoltaics $^{27}$, nanolithography ${ }^{28}$, sensing ${ }^{29}$, life sciences, and medical applications ${ }^{30}$ (see the more detailed discussion on the advantages of PMs in the Supplementary Information). Among the demonstrated metasurfaces, geometric metasurfaces (GMs), consisting of anisotropic meta-atoms with identical geometric parameters and spatially varying orientations, provide an orientation-controlled and dispersion-less phase control over the entire $2 \pi$ range for cross-circular polarization (CP) light $^{31}$. GMs are favorable in practical applications as

\section{(c) The Author(s) 2019}

\footnotetext{
(c) (i) Open Access This article is licensed under a Creative Commons Attribution 4.0 International License, which permits use, sharing, adaptation, distribution and reproduction in any medium or format, as long as you give appropriate credit to the original author(s) and the source, provide a link to the Creative Commons license, and indicate if changes were made. The images or other third party material in this article are included in the article's Creative Commons license, unless indicated otherwise in a credit line to the material. If material is not included in the article's Creative Commons license and your intended use is not permitted by statutory regulation or exceeds the permitted use, you will need to obtain permission directly from the copyright holder. To view a copy of this license, visit http://creativecommons.org/licenses/by/4.0/.
} 
they are naturally broadband, easy to design, robust to fabrication errors and variations in material properties, and are able to control transmission and reflection ${ }^{19,32-34}$.

Although controlling optical transmission is of interest for many applications, the operation efficiency of ultrathin transmission-type GMs is severely limited. On the one hand, in an ultrathin antenna, only the electric dipole (ED) can be excited. Symmetric forward and backward scattering of the ED imposes a 25\% theoretical upper limit on the cross-CP transmission, even when ignoring absorption losses ${ }^{35,36}$. Consequently, more than $75 \%$ of incident light is transmitted in the co-CP state, diffracted into undesired orders, or reflected. On the other hand, the notion that the metasurface efficiency can be increased by increasing the packing density of meta-atoms cannot be readily extended to GMs. At higher packing densities with smaller meta-atom interspaces, the near field of adjacent meta-atoms starts to couple and modifies the amplitude/ phase responses of individual meta-atoms to deviate from their designed values. Accordingly, developing an optimal design of GMs at a high packing density is a formidable task. Furthermore, for plasmonic GMs, the efficiency is further limited by the strong absorption in metals, particularly in the optical band. Consequently, the demonstrated efficiencies for transmission-type plasmonic GMs in the visible and near-infrared regions are $\leq 10 \%$ for realizing various functions ${ }^{37-40}$. Realizing efficient PMs in the visible and near-infrared range, thus, is of paramount technological importance ${ }^{41}$.

Meta-atoms that simultaneously support ED and magnetic dipole (MD), so-called Huygens meta-atoms, are able to break the scattering symmetry and enhance the transmission efficiency ${ }^{42,43}$. In a Huygens meta-atom, zero backscattering is realized when the ED and MD are of the same magnitude and phase, as their back-scattered radiations destructively interfere, which satisfies the first Kerker condition ${ }^{44}$. However, plasmonic Huygens metaatoms require metallic patterned multilayers, typically three layers or more ${ }^{36,45-48}$, which are difficult to fabricate, particularly when operating at short-wavelength optical range. In addition, previously reported Huygens meta-atoms are either isotropic or only capable of controlling linear polarization (LP) along a single direction. High-transmission GMs require the construction of a half-wave plate, in which the two orthogonal LP components of the incident CP light along the two main axes have a $\pi$-phase difference and maximum transmission ${ }^{31}$. The $\pi$-phase difference necessitates using anisotropic meta-atoms with nonoverlapping resonances at two orthogonal LPs, while maximum transmission requires satisfying the first Kerker condition at both LPs within the same wavelength range, which is difficult to achieve using anisotropic meta-atoms. The first Kerker condition can only be satisfied when the ED and $\mathrm{MD}$ are equal in magnitude and phase, which is a very restrictive condition, particularly for anisotropic meta-atoms. Consequently, increasing the transmission efficiency of GMs using the Huygens metasurface approach remains challenging. Other few-layer structures have been reported to have high transmission ${ }^{49,50}$. For example, by introducing two orthogonal gratings before and after a layer of metallic antennas, high-transmission PMs manipulating cross-LP light have been demonstrated in the terahertz ${ }^{51}$ and microwave ${ }^{52}$ regions. However, it is difficult to apply this three-layer scheme to GMs in the visible and nearinfrared regions. Until now, there has been no direct solution to address the low transmission of plasmonic GMs in the visible and near-infrared regions. A reflectiontype plasmonic GM reaching $80 \%$ efficiency in the visible and near-infrared regions has been reported by eliminating all the loss channels, except the inevitable absorption losses ${ }^{19}$. Ideally, a transmission-type plasmonic GM can reach an efficiency of $80 \%$ in the same wavelength region, if all undesired channels (i.e., reflection, co-CP transmission, and diffraction to undesired orders) are eliminated. Furthermore, absorption losses can be mitigated by designing the field distribution, as is shown in this work, or can be compensated by immersing the metasurface in a gain medium ${ }^{53}$.

In this work, we use multipole meta-atoms that support not only ED and MD but also an electric quadrupole (EQ) and a magnetic quadrupole (MQ) to construct an ideal half-wave plate. As shown in Fig. 1a, in multipole metaatoms, it is possible to completely suppress backscattering for both orthogonal polarizations by satisfying the so-called generalized Kerker condition ${ }^{54}$ and maintain the $\pi$-phase difference. This is the design principle of our work to be elaborated in this paper. We propose a multipole meta-atom design consisting of a metallic nanoaperture and a metallic nanorod separated by a perforated dielectric layer. The dimension of the nanorod (Fig. 1b) and thus the multipole response (Fig. 1c) can be modified by introducing a small air gap between the nanorod and the dielectric spacer separating adjacent meta-atoms. Tuning the meta-atom response allows us to simultaneously suppress backscattering for orthogonal LPs while maintaining an $\sim \pi$-phase difference. Furthermore, we dramatically mitigate inter-meta-atom crosstalk and reduce metallic absorption due to confining the field within the introduced air gap, as shown in Fig. 1d (more detail will be given later). Based on the multipole metaatom design, we demonstrate a transmission-type plasmonic GM with a theoretical efficiency of up to $45.5 \%$ at visible wavelengths. The ease of fabrication and high tolerance to fabrication errors of our multipole plasmonic metasurface (MPM) allowed us to experimentally achieve a transmission efficiency of $42.3 \%$ at $744 \mathrm{~nm}$. We developed a beam deflector with an experimental transmission 


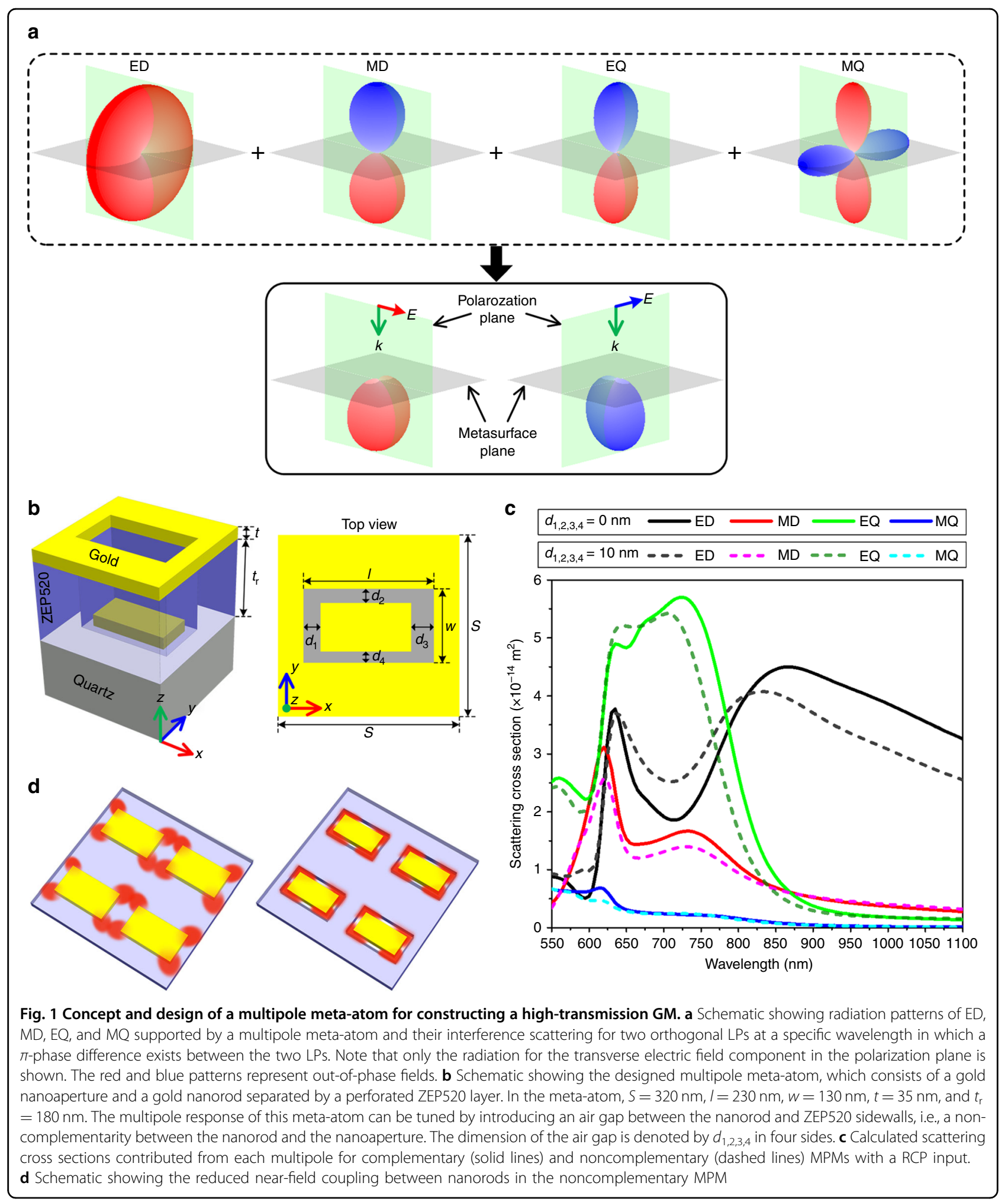

efficiency of $38.2 \%$ and extinction ratio (ER) of $7.8 \mathrm{~dB}$ over the co-CP transmission, which are $\sim 400 \%$ and tenfold improvements, respectively, over those of the stateof-the-art plasmonic GMs in the visible and near-infrared regions (see Table 1). In addition, we demonstrate a hologram with a $37 \%$ transmission efficiency, which also represents a fourfold improvement over PM-based holograms ${ }^{39}$. 
Table 1 Summary of previously reported transmissive geometric plasmonic metasurfaces in the visible and nearinfrared regions. NS: not specified

\begin{tabular}{|c|c|c|c|c|c|}
\hline Structure & Function & Wavelength (nm) & Efficiency & Extinction ratio & Reference \\
\hline Nanorod & Deflector and vortex generator & $670-1100$ & $<10 \%$ according to ref. ${ }^{38}$ & $-17 \mathrm{~dB}$ according to ref. ${ }^{16}$ & 5 \\
\hline Nanorod & Metalens & 740 & $5 \%$ & $<0 \mathrm{~dB}(\mathrm{NS})$ & 7 \\
\hline Nanorod & Hologram and metalens & 880 & $5 \%$ & $<0 \mathrm{~dB}(\mathrm{NS})$ & 10 \\
\hline V-shaped & Vortex generator & 780 & $3 \%$ & $\sim-10 \mathrm{~dB}$ & 13 \\
\hline V-shaped & Vortex generator & 780 & $8.6 \%$ & $\sim-10 \mathrm{~dB}$ & 14 \\
\hline Split ring & Vortex generator & 900 & $\sim 5 \%$ & $\sim-10 \mathrm{~dB}$ & 15 \\
\hline Nanoaperture & Vortex generator & 632.8 & $3.3 \%$ & $-4 \mathrm{~dB}$ & 16 \\
\hline Nanorod & Hologram & 810 & $4.5 \%$ & $<0 \mathrm{~dB}(\mathrm{NS})$ & 20 \\
\hline Nanorod & Polarization characterization & 940 & $7.6 \%$ & $<0 \mathrm{~dB}(\mathrm{NS})$ & 55 \\
\hline Nanorod and nanoaperture & Deflector and hologram & 744 & $38.2 \%$ & $7.8 \mathrm{~dB}$ & This work \\
\hline
\end{tabular}

\section{Results}

\section{Concept and design}

In GMs, the transmission coefficient of cross-CP light is expressed as $t_{\text {cross }-C P}=\frac{t_{x}-t_{y}}{2} e^{ \pm i 2 a 31}$, where + holds for a left circular polarization (LCP) input and - holds for a right circular polarization (RCP) input, $\alpha$ is the orientation angle of the meta-atom with respect to the $x$-axis, and $t_{x}$ and $t_{y}$ are the transmission coefficients for LP inputs along the $x$-axis and $y$-axis, respectively, when $\alpha=0$. Thus, to maximize the cross-CP transmittance $\left(T_{\text {cross-CP }}\right)$, which is proportional to $\left|t_{\text {cross-CP }}\right|^{2}$, the main goal is to realize phase $\left(t_{x}\right)$-phase $\left(t_{y}\right) \sim \pi$ and high $\left|t_{x, y}\right|$. For lossless or low-loss GMs, an ideal meta-atom is a half-wave plate with zero reflection at both LPs. After designing a meta-atom with high $T_{\text {cross-CP }}$, an efficient GM also requires that the metaatom supports phase responses solely controlled by its orientation and nearly identical amplitude responses for different orientations. To meet this condition, the coupling between adjacent meta-atoms should be negligible, as adjacent coupling can modify the meta-atom's amplitude/ phase response due to hybridization.

As mentioned earlier, based on the Huygens meta-atom approach, which depends on the destructive interference of the back-scattered light from the ED and $\mathrm{MD}^{44,56}$, it is difficult to simultaneously satisfy the $\pi$-phase-difference and zero-reflection conditions. To overcome this problem, we use meta-atoms that support multipole moments. As shown in Fig. 1a, zero reflection for both LPs is possible if the multipole moments destructively interfere in the backward direction, which leads to the definition of a generalized Kerker condition ${ }^{54,57}$. Figure $1 \mathrm{~b}$ shows the design of a multipole meta-atom, which is composed of a rectangle-shaped gold nanoaperture and a gold nanorod separated by a perforated dielectric resist layer with an etched hole. The nanorod is situated in the etched hole. The nanoaperture has the same dimension as the etched hole, while the dimension of the nanorod can be tuned to be smaller, i.e., non-complementarity between the nanorod and nanoaperture can be introduced by introducing an air gap between the nanorod and the sidewall of the etched hole. The dimensions of the air gap are represented by the gap sizes on the four sides, which are defined as $d_{1}, d_{2}, d_{3}$, and $d_{4}$. The separation between the two gold layers is $t_{\mathrm{r}} . S$ is the subperiod of the metaatom. $l$ and $w$ are the length and width of the nanoaperture, respectively. $t$ is the gold thickness. Unless otherwise stated, $S=320 \mathrm{~nm}, l=230 \mathrm{~nm}, w=130 \mathrm{~nm}, t$ $=35 \mathrm{~nm}$, and $t_{\mathrm{r}}=180 \mathrm{~nm}$ in all calculations.

To uncover the importance of tuning the multipole excitations with the air gap, i.e., non-complementarity, we study a complementary meta-atom design without an air gap, i.e., $d_{1,2,3,4}=0 \mathrm{~nm}$, and a noncomplementary design with a simple symmetric air gap of $d_{1,2,3,4}=10 \mathrm{~nm}$. By using an exact multipole expansion approach ${ }^{58}$, we can calculate the multipole response of these meta-atoms. Figure 1c shows the calculated scattering cross sections of the ED, MD, EQ, and MQ in complementary (solid lines) and non-complementarity (dashed lines) meta-atoms, which indicates that both meta-atoms sustain multipole resonances in the visible and near-infrared regions. In particular, the high-order EQ is comparable or even stronger than the ED and MD; therefore, it contributes to the multipole interference. More importantly, the multipole responses, and correspondingly, their interference are tunable by introducing non-complementarity. As shown later, this tunability can facilitate destructive interference in the backward direction, i.e., satisfying the generalized Kerker condition.

Another advantage of the noncomplimentary metaatom is that by introducing non-complementarity the 
near field of the nanorod is strongly confined within the air gap instead of the dielectric spacer and metallic nanorod (Fig. 1d). This result is a consequence of the continuity of the displacement field normal component, which results in a higher-field intensity for regions with lower electric permittivity, e.g., air, and is analogous to hybrid plasmonic structures ${ }^{59}$. The reduced field inside the resist spacer minimizes the near-field coupling between nanorods in adjacent meta-atoms, which allows an increase of the meta-atom packing density and hence the metasurface efficiency. Furthermore, the reduced field inside the nanorod decreases metallic absorption, which further increases the efficiency.

For GMs, we are concerned with the satisfaction of the generalized Kerker condition for two orthogonal LPs and the phase difference between them. We define the generalized Kerker coefficients as ${ }^{57}$

$$
\begin{aligned}
K_{x} & =\left(1+r_{a s} e^{i 2 k z_{0}}\right)\left(p_{x}-\frac{i k}{2 c} M_{y z}\right) \\
& -\left(1-r_{a s} e^{i 2 k z_{0}}\right)\left(\frac{m_{y}}{c}-\frac{i k}{6} Q_{x z}\right)
\end{aligned}
$$

for the $x$-LP input along the $-z$ direction and

$$
\begin{aligned}
K_{y} & =\left(1+r_{a s} e^{i 2 k z_{0}}\right)\left(p_{y}+\frac{i k}{2 c} M_{x z}\right) \\
& -\left(1-r_{a s} e^{i 2 k z_{0}}\right)\left(\frac{m_{x}}{c}+\frac{i k}{6} Q_{y z}\right)
\end{aligned}
$$

for the $y$-LP input along the $-z$ direction, where $r_{\mathrm{as}}=$ $\left(1-n_{\mathrm{s}}\right) /\left(1+n_{\mathrm{s}}\right)$ is the reflection coefficient from the air cladding to a substrate with a refractive index $n_{\mathrm{s}}, z_{0}$ is the distance between the multipole expansion center and the substrate interface, and $k$ and $c$ are the wavenumber and light speed in air, respectively. $p, m, Q$, and $M$ are the ED, $\mathrm{MD}, \mathrm{EQ}$, and MQ moments, respectively. The backscattered far field from a meta-atom is proportional to $\backslash \mathrm{cr} K_{x, y}$. Accordingly, the generalized Kerker condition for zero reflection is fulfilled when $K_{x, y}=0^{57}$.

Figures $2 \mathrm{a}-\mathrm{c}$ show the calculated generalized Kerker coefficients $\left(K_{x, y}\right)$, transmittances $\left(T_{x, y}\right)$, and phase of $t_{x, y}$, respectively, at two LPs for complementary and noncomplementary meta-atoms. The calculations of $p, m, Q$, and $M$ at two LPs are given in the Supplementary Information (SI) Fig. S1. Clearly, the local minima of $K_{x, y}$, i.e., where the meta-atom approaches the generalized Kerker condition, correspond to the transmission peaks for $T_{x, y}$ in all cases. For the complementary design, $K_{x}$ is minimum at $\lambda \sim 700 \mathrm{~nm}$ and $K_{y}$ is minimum at $\lambda \sim 800 \mathrm{~nm}$, corresponding to transmission peaks for $T_{x}$ of $\sim 59 \%$ and $T_{y}$ of $\sim 45 \%$, respectively. Between the two transmission peaks, the metasurface has a high $T_{x}$ and $T_{y}$. At the same time, the phase difference between $t_{x}$ and $t_{y}$ is $\sim \pi$ in the same wavelength range, which makes the design behave as an efficient half-wave plate in the transmission direction, with a maximum $T_{\text {cross-CP }}$ of $35.2 \%$ at $\lambda \sim 760 \mathrm{~nm}$ (Fig. $2 \mathrm{~d}$ ), i.e., with an efficiency $10 \%$ higher than the $25 \%$ limit for ultrathin metasurfaces. The calculation described in SI Fig. S2 shows that the meta-atom works as an efficient half-wave plate in the reflection direction as well at the same wavelength, with a cross- $\mathrm{CP}$ reflection efficiency of $37.7 \%$. In both directions, the unwanted co-CP lights are $<1 \%$. Accordingly, the designed complementary MPM can realize a combined function of a half-wave plate and a 50:50 beam splitter with a net cross-CP conversion efficiency of $73 \%$, which is close to that of the state-of-the-art PMs operating in the reflection mode ${ }^{19}$ and dielectric metasurfaces operating in the transmission mode ${ }^{32-34}$.

However, in most applications, wavefront control of the transmitted light with high efficiency is preferred. In our design, the introduction of non-complementarity between the nanoaperture and nanorod is the key to engineering the multipole interference and enhancing the $T_{\text {cross-CP. }}$ As shown in Figs. 2a, b and Fig. S1, the effect of introducing non-complementarity on the multipole excitations of the $x$-LP input is almost negligible in the wavelength range of interest. However, for the $y$-LP input, the noncomplementarity results in a reduced $K_{y}$ and enhanced $T_{y}$ within the wavelength range of interest, i.e., the wavelength range where $T_{x}$ is maximum. The corresponding $y$-LP transmission peak is increased to $T_{y} \sim 71 \%$ at $780 \mathrm{~nm}$, which is closer to the peak wavelength of $T_{\mathrm{x}}$ at $705 \mathrm{~nm}$ (see the multipole radiations and their interferences at $705 \mathrm{~nm}$ for $x$-LP and $780 \mathrm{~nm}$ for $y$-LP in SI Fig. S3). Meanwhile, a phase difference of $\sim \pi$ is still maintained (Fig. 2c). As a result, we achieve an overall enhancement in the $T_{\text {cross-CP }}$ of the noncomplementary design (Fig. 2d). The peak efficiency $\left(E_{\mathrm{p}}\right)$ is increased to $45.5 \%$ at a peak wavelength $\left(\lambda_{\mathrm{p}}\right)$ of $751 \mathrm{~nm}$, i.e., $T_{\text {cross-CP }}$ is increased by $>10 \%$. Here, we note that the $26 \% T_{y}$ increase is not only due to approaching the generalized Kerker condition, which decreases the reflection by $14 \%$, but also due to a reduction in absorption at the transmission maximum of $\sim 12 \%$ (SI Fig. S4).

The reduced near-field coupling between the metaatoms after introducing non-complementarity is evident in Figs. 2e, f, which shows the calculated phase and amplitude $(\lambda=750 \mathrm{~nm})$ of $t_{\text {cross-CP }}$, respectively, as a function of the orientation angle $(\alpha)$ for complementary and noncomplementary meta-atoms (see the broadband calculation in SI Fig. S5). Both designs support good orientation-controlled phase responses. However, the $t_{\text {cross-CP }}$ amplitude of the complementary design significantly changes as a function of $\alpha$, reaching a minimum at an $\alpha$ of $\sim 40^{\circ}$, where the meta-atom interspace is the minimum (SI Fig. S6), i.e., the near-field coupling is the maximum. On the contrary, the noncomplementary meta-atom has higher and relatively flat amplitudes. 


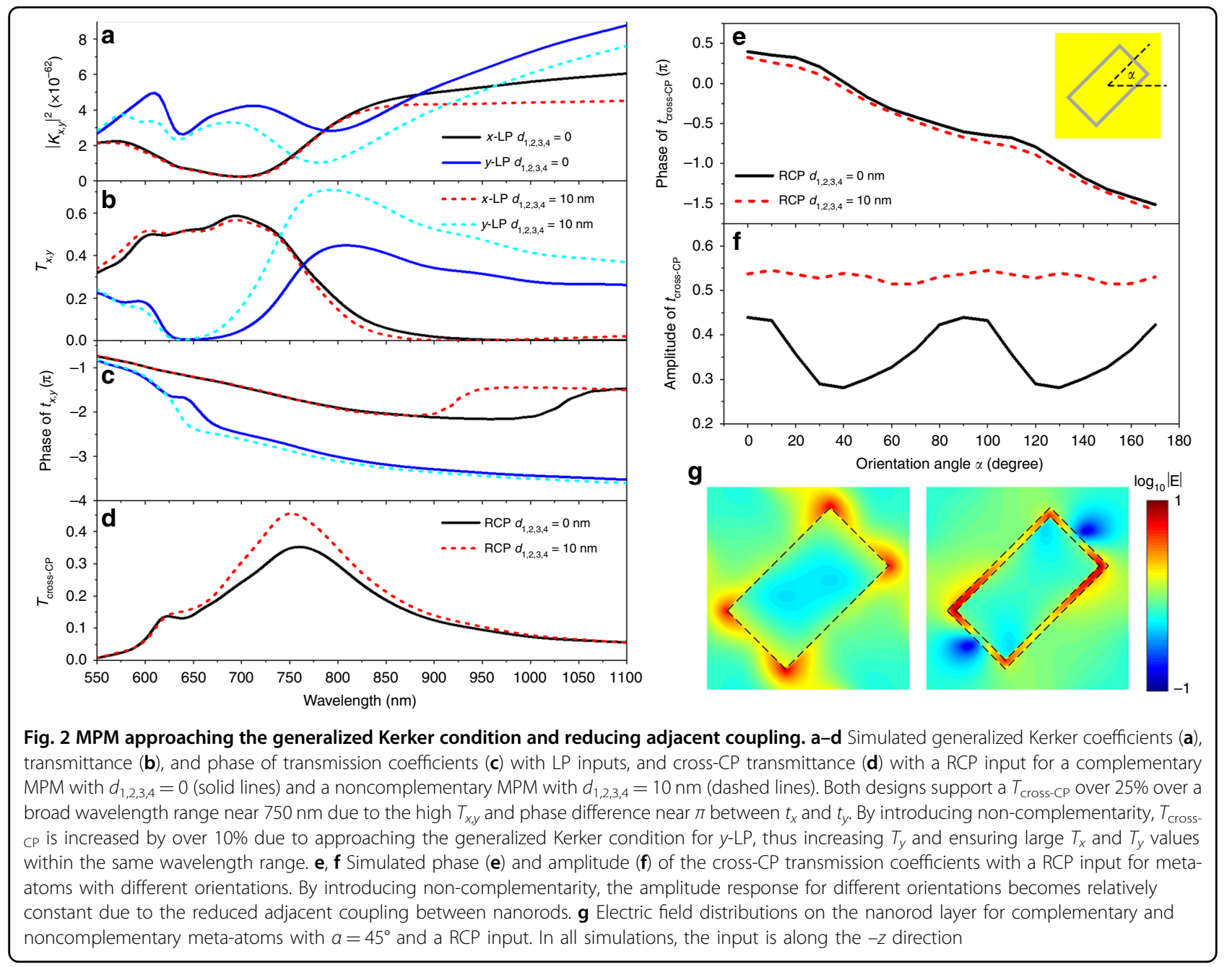

Figure $2 \mathrm{~g}$ shows the electric field distributions of the nanorod layer for complementary (left) and noncomplementary (right) meta-atoms with $\alpha=45^{\circ}$. The localized field inside the air gap mitigates the crosstalk between noncomplementary meta-atoms. The reduced coupling of noncomplementary meta-atoms allows further increases of the efficiency by using a higher packing density, while for the complementary design, the efficiency decreases (SI Fig. S7). Note that meta-atom crosstalk does not always degrade the performance of metasurfaces that do not adopt the GM scheme. For example, strong coupling has been deliberately designed to improve the cross-LP transmission in a V-shaped bilayer metasurface ${ }^{60}$. The adopted meta-atoms with a combination of solid and inverse structures share some of the aspects of our complementary design, but our work is mostly focused on a noncomplementary design. Furthermore, a strong-coupling design will always impose complex designs and stringent requirements on the fabrication accuracy and cannot be used for GMs.

\section{Fabrication and tolerance}

The noncomplementary design can be realized with the facile fabrication method described in Fig. 3a. First, rectangle-shaped patterns were defined on the ZEP520 resist layer by electron beam lithography (EBL). Then, a thin gold layer was deposited by electron beam evaporation. The noncomplementary nanorod was realized by tilting the stage by a small angle and halting the stage rotation. To realize $d_{2,3}=20 \mathrm{~nm}$ for the MPM with $\alpha=0^{\circ}$, a small tilt with an azimuthal angle $\phi=45^{\circ}$ and a polar angle $\theta \approx 9^{\circ}$ was applied. To avoid gold deposition on the resist sidewalls, a dimension bias and a high dose were applied during the EBL to achieve a slight undercut profile of the resist sidewalls ${ }^{61}$. This fabrication procedure is simpler than that for single-layer PMs, which requires an additional step to remove unwanted metals by a lift-off or etch-back process" ${ }^{62}$ (see the section "Materials and methods" for more details). Note that when adopting the tilt deposition technique shown in Fig. 3a, $d_{1}$ and $d_{4}$ will be zero. However, even when $d_{1,4}=0$, one can see from 

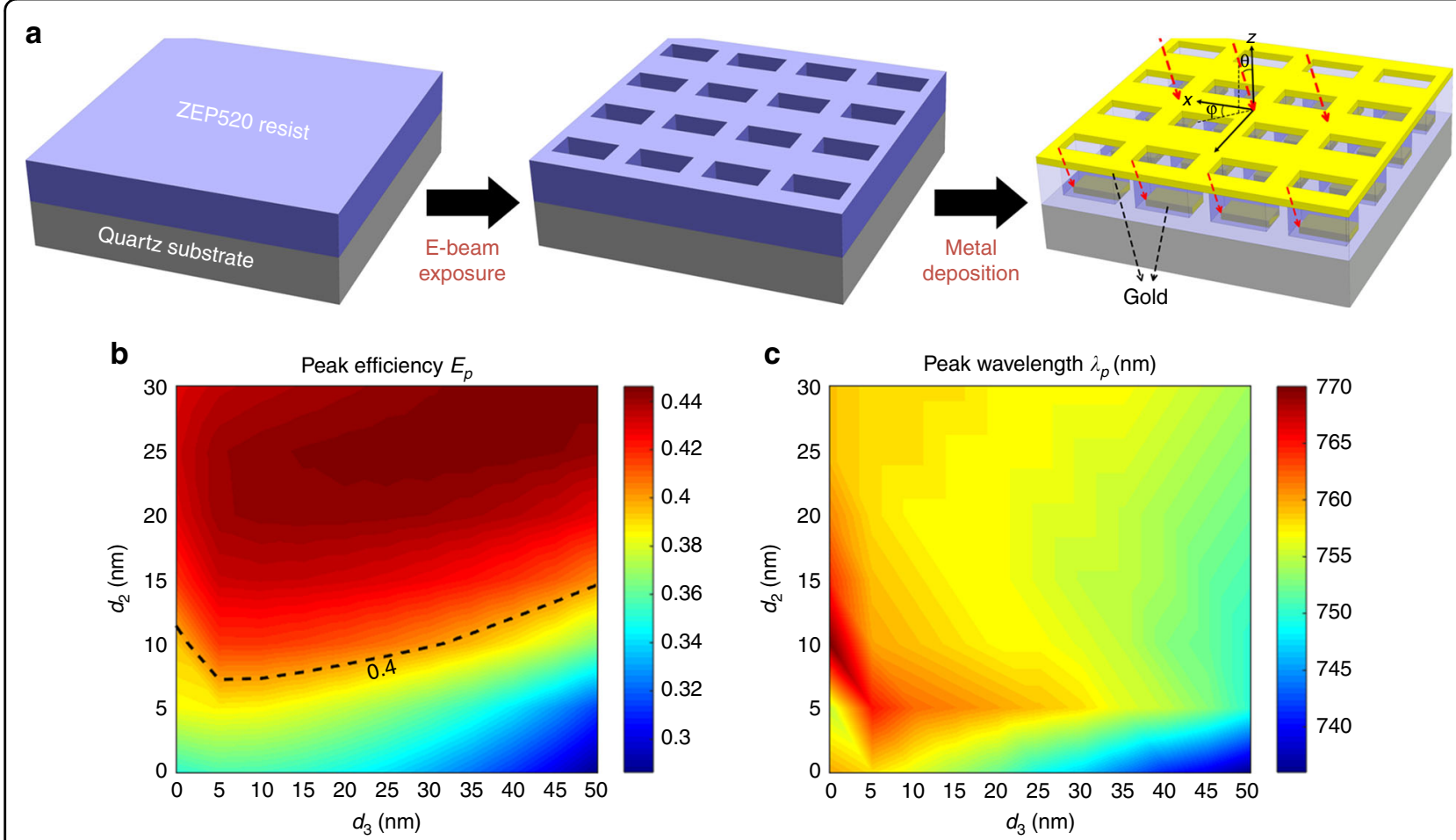

Fig. 3 Fabrication method and high fabrication tolerance. a Fabrication procedure of the noncomplementary MPM with $d_{1,4}=0$ and $d_{2,3}=$ $20 \mathrm{~nm}$, in which a tilt metal deposition technique is utilized. The red dashed arrows indicate the metal deposition direction. In this case, the nanorod dimension mismatch values are expressed by $d_{1,4}=0, d_{2}=t_{r} \tan \theta \sin \varphi$, and $d_{3}=t_{r} \tan \theta \cos \varphi$. $\mathbf{b}, \mathbf{c}$ Simulated transmission efficiency maximum $E_{\mathrm{p}}$ (b) and related wavelength $\lambda_{p}$ (c) of the noncomplementary MPM as functions of $d_{2}$ and $d_{3}$ when $d_{1,4}=0$. The black dashed line in (b) marks the contour of 0.4 . $E_{\mathrm{p}}$ is higher than $40 \%$ at visible wavelengths for a wide range of mismatch values

Fig. 3b that $E_{\mathrm{p}}$ maintains high values $(>40 \%)$ for a wide range of values of $d_{2}$ and $d_{3}$. Meanwhile, $\lambda_{\mathrm{p}}$ remains within the wavelength range of interest, as shown in Fig. 3c. Figures $3 b-c$ highlight the high tolerance of our design to fabrication errors. The performance of the noncomplementary design shows high tolerance to other parameters as well (SI Figs. S8 and S9). Because a large deposition tilt may cause metal deposition on the sidewalls and an observable chirality, we use a relatively small air gap with $d_{1,4}=0$ and $d_{2,3}=20 \mathrm{~nm}$. Such an asymmetric noncomplementary design supports the multipole response as well and a theoretical $E_{\mathrm{p}}$ of $44.3 \%$ at $757 \mathrm{~nm}$ (SI Fig. S10), which provides a similar efficiency improvement to the symmetric case with $d_{1,2,3,4}=10 \mathrm{~nm}$. The complementary design is obtained by enabling stage rotation during deposition.

\section{Demonstration of efficient cross-CP transmission}

Figure 4a shows scanning electron microscopy (SEM) images of the fabricated noncomplementary MPM with $d_{1,4}=0$ and $d_{2,3}=20 \mathrm{~nm}$. The measured and calculated $T_{x, y}$ are shown in Fig. $4 \mathrm{~b}$, and they are in good agreement. $T_{y}$ is experimentally found to be $\sim 68 \%$ at $790 \mathrm{~nm}$, which indicates the efficient suppression of back-scattered $y$-LP light. The details of the measurement setup are provided in the "Materials and methods" and SI Fig. S19. Figure 4c shows the $T_{\text {cross-CP }}$ with the RCP input. The black solid, red dashed, and blue dash-dotted lines represent the experimental result, simulation of noncomplementary MPM, and simulation of complementary MPM, respectively. The demonstrated $T_{\text {cross-CP }}$ exceeds $10 \%$ over a wide wavelength range of $630-970 \mathrm{~nm}$. In particular, $E_{\mathrm{p}}$ is $42.3 \%$ at $744 \mathrm{~nm}$. The measured $E_{\mathrm{p}}$ is larger than the simulation value of the complementary design $(35.2 \%$ at $760 \mathrm{~nm}$ ) by $\sim 7 \%$ but lower than the simulation value of the noncomplementary design $(44.3 \%$ at $757 \mathrm{~nm})$ by $\sim 2 \%$. This small deviation is likely due to the chromium adhesion layer and a slightly tilted edge surface of the metal layer, which are omitted in the simulation. Similar results of $T_{\text {cross-CP }}$ for the LCP input are obtained (SI Fig. S11). The noise in the measured $T_{\text {cross-CP }}$ below $630 \mathrm{~nm}$ is due to the bandwidth limitation of the quarter-wave plate used in the experiment (see the section "Materials and methods").

\section{Application in beam deflection}

By introducing a phase gradient on the surface, we test our MPM as a beam deflector. Figure 5 a shows a SEM 

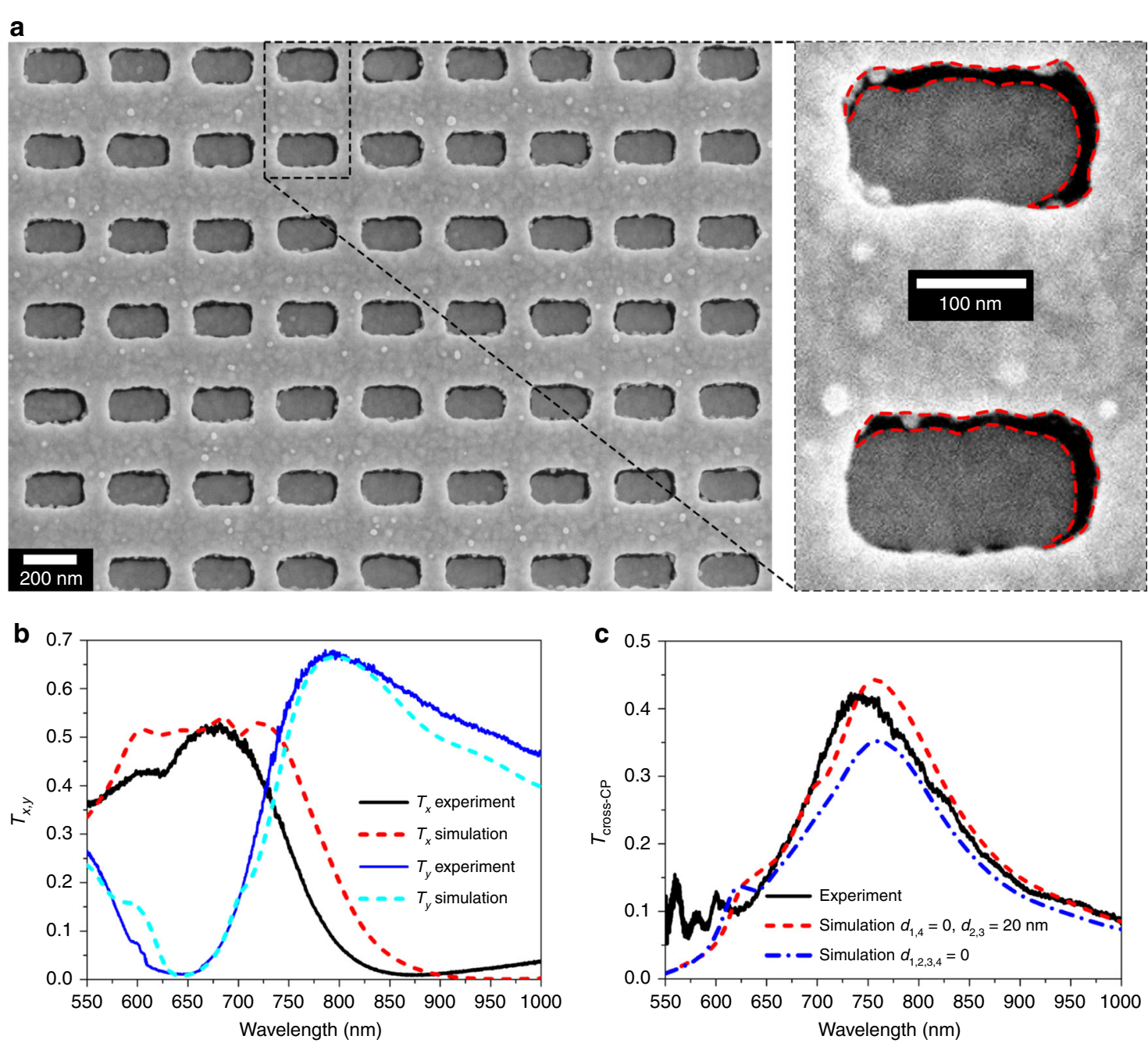

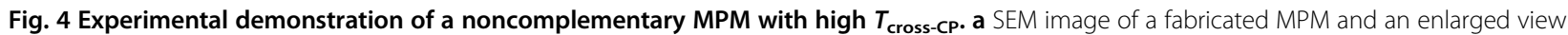
of two meta-atoms, in which asymmetric air gaps are clearly observable. The red dashed lines mark the edges of the air gaps. $\mathbf{b}$, c Simulated and measured LP (b) and cross-CP (c) transmittances. The solid, dashed, and dash-dotted lines represent the experimental results, simulation of the noncomplementary MPM with $d_{1,4}=0$ and $d_{2,3}=20 \mathrm{~nm}$, and simulation of the complementary MPM, respectively. The measured efficiency is higher than that for the simulation of the complementary MPM by $\sim 7 \%$, confirming the role of tuning the multipole response in approaching the generalized Kerker condition

image of the fabricated GM and an enlarged view of one unit cell. A single unit cell consists of eight subunits (SUs) with an orientation step of $22.5^{\circ}$ (period: $\Lambda=2.56 \mu \mathrm{m}$ ). Note, the dimension mismatches (i.e., $\left.d_{1,2,3,4}\right)$ for SUs with different orientations differ during the tilted deposition. However, the high efficiency persists for each SU because it is relatively insensitive to the values of $d_{1,2,3,4}$, as we showed earlier (Figs. 3b, c and Fig. S8). The average mismatch values of each SU measured from the SEM image shown in Fig. $5 \mathrm{a}$ are used for the simulations. The detailed simulation structure is described in SI Fig. S12b. Figure $5 \mathrm{~b}$ shows the experimentally measured $T_{\text {cross-CP }}$ and $T_{\mathrm{Co}-\mathrm{CP}}$ as functions of the wavelength and output angle for the RCP input with a normal incidence, which agree with the corresponding simulation result presented in Fig. 5c. The black dashed lines represent the theoretical output angle of the anomalous beam, which is determined by $\arcsin (\lambda / \Lambda)^{27}$. Figure $5 \mathrm{~d}$ shows the measured beam deflection efficiency of the anomalous transmission and $\mathrm{ER}$, defined as $10 \times \log _{10}\left(T_{\text {cross-CP }} / T_{\text {co-CP }}\right)$, and the corresponding theoretical calculations for the noncomplementary and complementary designs. The theoretical (40.4\%) and experimental (38.2\%) $E_{\mathrm{p}}$ of the noncomplementary design are higher than the theoretical (20.7\%) $E_{\mathrm{p}}$ of the complementary design. In addition, the measured efficiency exceeds the $25 \%$ limit for ultrathin metasurfaces over the wide $690-810-\mathrm{nm}$ range. The measured ER peaks at $7.8 \mathrm{~dB}$ at $\sim 745 \mathrm{~nm}$ and exceeds $0 \mathrm{~dB}$ 


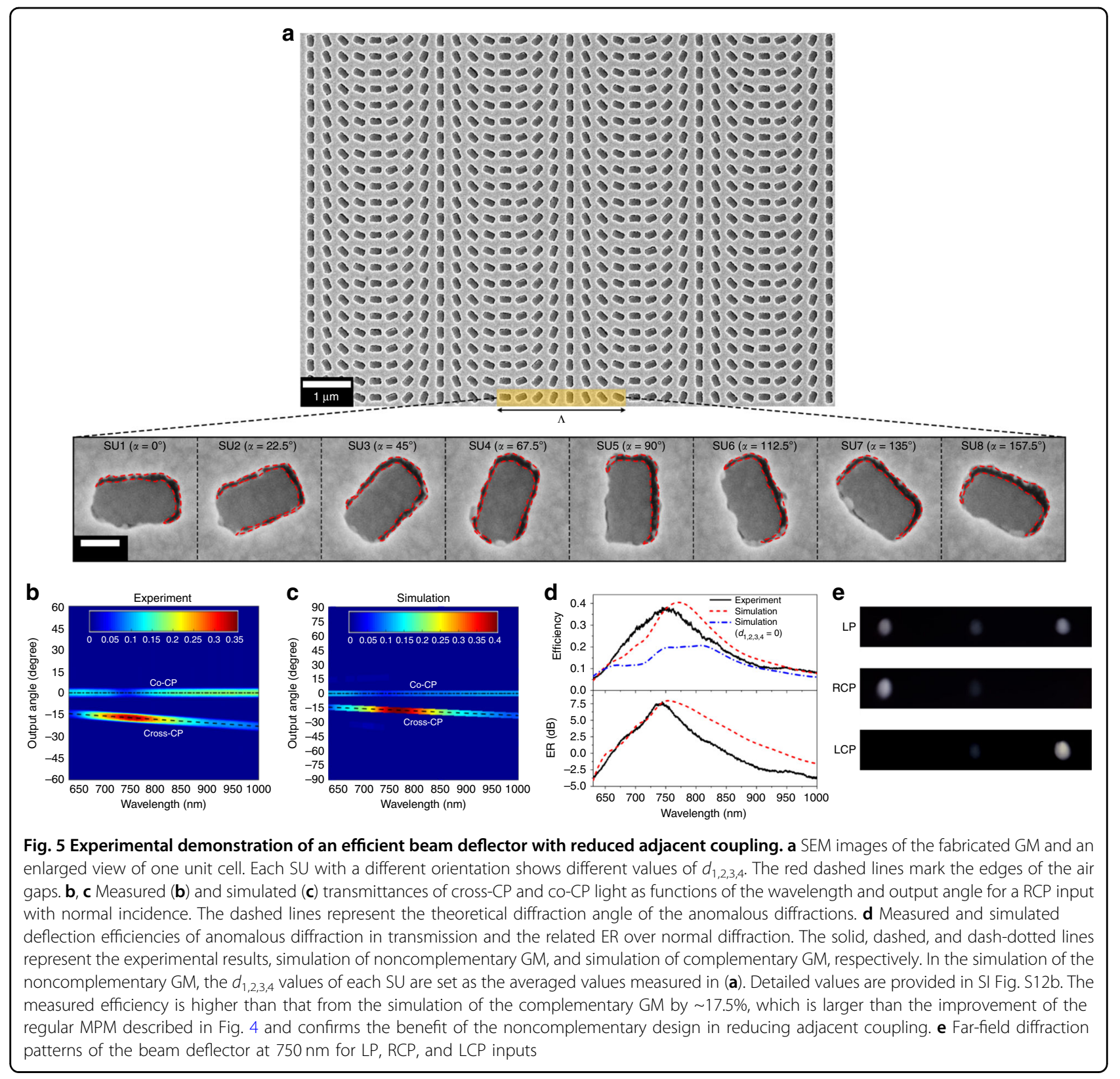

between 660 and $850 \mathrm{~nm}$. Both $E_{\mathrm{p}}$ and ER are significantly higher than the values of the current state-of-the-art plasmonic GMs $\left(E_{\mathrm{p}} \leq 10 \%, \mathrm{ER} \leq 0 \mathrm{~dB}\right)$ in the visible and near-infrared regions in realizing various functionalities $^{5,13,15,55}$. Furthermore, the close agreement between the theoretical simulation $\left(E_{\mathrm{p}}=40.4 \%, E R=8 \mathrm{~dB}\right)$ and experimental measurement $\left(E_{\mathrm{p}}=38.2 \%, \quad \mathrm{ER}=7.8 \mathrm{~dB}\right)$ indicates the tolerance of our design to fabrication errors. The beam deflection results for the LCP input are very similar (SI Fig. S13).

We note that the experimental $E_{\mathrm{p}}$ of the noncomplementary MPM was $\sim 7 \%$ larger than the theoretical $E_{\mathrm{p}}$ of the complementary design shown in Fig. 4c due to approaching the generalized Kerker condition. However, the experimental $E_{\mathrm{p}}$ of the noncomplementary $\mathrm{GM}$ is $\sim 17.5 \%$ larger than the theoretical $E_{\mathrm{p}}$ of the complementary GM design. This additional enhancement in the transmission efficiency benefits from reducing the near-field coupling of the meta-atoms. In GMs, nonuniform amplitude responses resulting from coupling-induced modifications can decrease the total transmittance and increase the portion of the transmitted power into undesired diffraction orders. The reduced coupling between meta-atoms is shown by the relatively flat-amplitude responses of eight SUs and the electric field distributions in one unit cell of both complementary and noncomplementary GMs at 

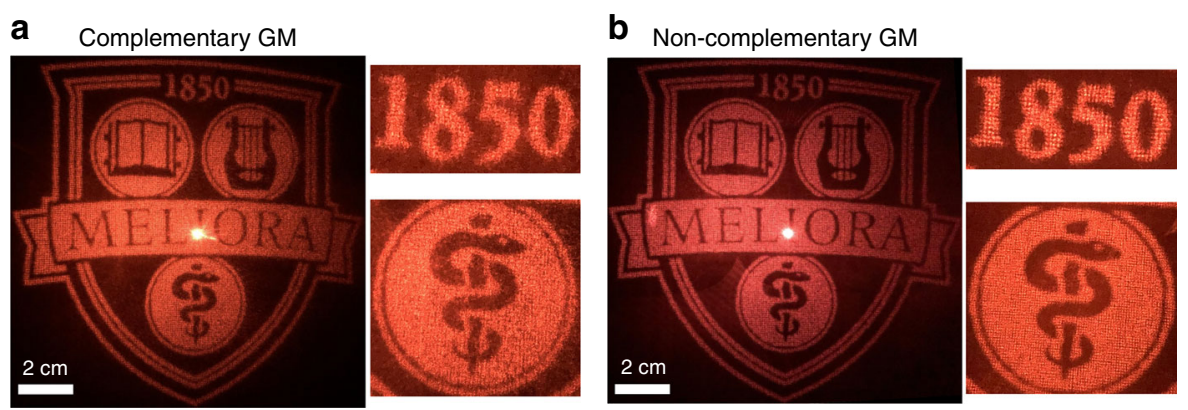

Fig. 6 Experimental demonstration of a hologram. a, b Holography images at $750 \mathrm{~nm}$ for the complementary GM (a) and noncomplementary GM (b). The enlarged views clearly show the better quality of the holography images obtained with the noncomplementary GM

$\lambda=750 \mathrm{~nm}$ in Fig. S12. The simulations described in SI Figs. S14 and S15 show that diffractions to undesired high orders for the noncomplementary GM are less than onetenth of the zero order for both cross-CP and co-CP. In the experiment, the high-order diffractions are within the noise level of the spectrometer.

Figure 5e shows the far-field diffraction patterns at $750 \mathrm{~nm}$ with the LP, RCP, and LCP inputs (see SI Fig. S16 for the results at various wavelengths). The central spots and the spots on each side represent co-CP normal diffraction and cross-CP anomalous diffraction beams, respectively. In contrast to previous experiments using single-layer $\mathrm{PMs}^{3-5,15,55,63}$, the anomalous diffracted beams from the GM deflector are stronger than the normal beams, which is consistent with the broadband experiments described in Figs. 5b and S13b.

\section{Application in a hologram}

We demonstrate the versatility of the proposed design by realizing a record-high efficiency hologram using a plasmonic GM. The phase pattern of the target image, the logo of the University of Rochester, is computed by means of the Gerchberg-Saxton phase-retrieval algorithm $^{64}$. Figure 6 shows experimental holography images in the far field at $\lambda=750 \mathrm{~nm}$. Figures $6 \mathrm{a}$ and $6 \mathrm{~b}$ are obtained from complementary and noncomplementary plasmonic GMs, respectively. While both designs show a clear reconstruction of the target image, the noncomplementary design offers a higher transmission efficiency and clearer details when looking at the enlarged view. This improvement is due to the aforementioned efficiency enhancement and smaller amplitude modification from the reduced adjacent coupling. The hologram efficiencies are measured to be $26.7 \%$ and $37 \%$ for the complementary and noncomplementary designs, respectively. These results are also better than those of the state-of-the-art GMs in the visible and nearinfrared regions ${ }^{39}$. The hologram is broadband as well, as we demonstrate in SI Fig. S17, which shows holography images at various wavelengths. The bright spots in the center of the reconstructed images arise from the zero-order light not coupling into the metasurface due to beam overfilling.

\section{Discussion}

A significant increase in the transmission efficiency of plasmonic GMs is facilitated by tuning the multipole response of individual meta-atoms and by minimizing the crosstalk between meta-atoms. The maximum efficiency of the MPM is on the long-wavelength side of the visible spectrum, $744 \mathrm{~nm}$. However, the demonstrated high efficiency exceeds that of the state-of-the-art GMs over a wide wavelength range of $630-970 \mathrm{~nm}$, i.e., in the redcolor range of the visible (visible covers $380-780 \mathrm{~nm}^{65}$ ) and near-infrared regions. This concept and these techniques can be used for shorter visible wavelengths (see a design in SI Fig. S18) or longer-wavelength regions by tuning the dimensions of the meta-atom. This concept can be used for dielectric metasurfaces as well. The generalized Kerker condition, in principle, allows for perfect transmission, reflection, or absorption ${ }^{54}$. Accordingly, the transmission efficiency can be increased further. By eliminating all co- $\mathrm{CP}$ transmissions and co- $\mathrm{CP}$ and cross$\mathrm{CP}$ reflections with further optimization, it is possible to enhance the transmission efficiency to $>70 \%$ and ensure that it is only limited by the intrinsic absorption losses in metals. Absorption can be mitigated by storing the field inside an introduced air gap, as we demonstrated, or by using metals with lower Ohmic losses. For PMs operating at longer wavelengths beyond the IR range with low absorption losses, it is possible to achieve almost $100 \%$ transmission with a MPM, which is simpler to fabricate compared with transmit arrays and multilayer metasurfaces. Furthermore, reflective-type metasurfaces can benefit from the generalized Kerker, no-front-scattering condition. On the other hand, perfect and selective light absorption can be achieved by eliminating both reflection and transmission, using the generalized Kerker approach. In the future, even higher-order multipoles can also be included to improve the performance of $\mathrm{PMs}^{66,67}$. 


\section{Materials and methods Fabrication}

Fabrication was carried out at the Cornell Nanoscale Facility. The metasurface was fabricated on a 500- $\mu \mathrm{m}$ thick quartz wafer from Mark Optics at Santa Ana, CA, USA. After cleaning with acetone and isopropyl alcohol, a layer of positive electron beam resist ZEP520 with a thickness of $180 \mathrm{~nm}$ was spin-coated on the substrate, followed by baking for $2 \mathrm{~min}$ at $170^{\circ}$ on a hot plate. Then, a thin layer of conductive polymer Espacer was spincoated to dissipate charge in the EBL process. Next, rectangle-shaped nanostructure patterns were defined on the resist layer by EBL on a JEOL 9500 system $(100 \mathrm{kV}$, $1 \mathrm{nA})$. The patterned area was $400 \times 400 \mu \mathrm{m}$ for the deflector and $655 \times 655 \mu \mathrm{m}$ for the hologram. A dimension bias $(25 \mathrm{~nm})$ and a relatively high dose $\left(380 \mu \mathrm{C} / \mathrm{cm}^{2}\right)$ were applied to achieve a slight undercut profile of the resist. After EBL, the Espacer was removed by water rinsing. Low-temperature development with high contrast was implemented by successively developing the sample in ZED-N50 for $45 \mathrm{~s}$, in methyl isobutyl ketone for $30 \mathrm{~s}$, and in isopropyl alcohol for $30 \mathrm{~s}$. The sample was then dried by a nitrogen blow. After development, $3 \mathrm{~nm}$ of chromium and $32 \mathrm{~nm}$ of gold were deposited in an electron beam evaporation system (Kurt J. Lesker PVD 75) that had a stage that could be tilted and rotated. In the tilt deposition, there was no metal deposition on the bottom layer in the shadow area of the resist. Theoretically, for a deposition direction along $-(\sin \theta \cos \phi, \sin \theta \sin \phi, \cos \theta)$ determined by the azimuthal $(\phi)$ and polar $(\theta)$ angles, the shadow dimensions (i.e., mismatch values) can be expressed by $t_{\mathrm{r}} \tan \theta|\sin (\phi-\alpha)|$ and $t_{\mathrm{r}} \tan \theta|\cos (\phi-\alpha)|$ in two directions for a meta-atom with an orientation angle $\alpha$. In the fabrication of noncomplementary MPM and GM, the sample rotation was disabled, and a small sample tilt of $\phi=45^{\circ}$ and $\theta \approx 9^{\circ}$ was applied during deposition. In the fabrication of the complementary GM for the hologram, stage rotation was enabled. No liftoff was processed after deposition. The fabricated metasurface was characterized by a scanning electron microscope (Hitachi S4100). All the SEM images were taken at normal incidence to the sample surface.

\section{Simulation}

Simulations were performed using the commercially available finite-difference time-domain software from Lumerical. In the simulations, LP or CP light was injected from the top in air. The CP input source and spin state were realized by adding two linear polarized sources in the $x$ and $y$ directions and setting the initial phase between them to $\pm \pi / 2$. The periodic and perfectly matched-layer boundary conditions were applied along the transverse and longitudinal directions with respect to the propagation of light. The dielectric constants of the gold and quartz substrates were directly obtained by using the Gold-Johnson and Christy database and the $\mathrm{SiO}_{2}$ Palik database in the software material's library, respectively. The refractive index of ZEP520 resist was obtained from spectroscopic ellipsometry measurements.

\section{Measurement}

The measurement setups are shown in SI Fig. S19. In the broadband measurements, a collimated beam from a broadband lamp source (Thorlabs: SLS301, wavelength range: $360-2700 \mathrm{~nm}$ ) was focused onto the sample, which was mounted on a three-dimensional moveable and rotatable stage. Before the focusing lens, a broadband Glan-Thompson polarizer (Melles Griot: 03 PTH 112/C, wavelength range: $350-2300 \mathrm{~nm}$ ) and a quarter-wave plate (Thorlabs: AQWP10M-980, wavelength range: 630-1200 nm) were utilized to generate CP. The transmitted beam was collected by an objective $(\times 10,0.25)$. The spin state of the output was selected by another broadband quarter-wave plate and polarizer set. The quarter-wave plates were only inserted into the setup during the $\mathrm{CP}$ measurements. The beam was finally focused into the fiber collector of a spectrometer (Photon Control: SPM001, wavelength range: 300-1000 nm). An iris was used to select the part of the light that passed through the metasurface area. Therefore, this setup was able to measure the LP and CP transmissions of the metasurface over wavelength ranges of 360-1000 and 630-1000 nm, respectively. The transmittance of the metasurface was normalized to the transmittance through the quartz substrate only, which was $~ 93 \%$ in the studied wavelength range.

In the CW deflection and hologram experiments, a CW output at $730-800 \mathrm{~nm}$ was obtained from a Griffin $\mathrm{Ti}$-Sapphire femtosecond laser (KM-Labs, Inc.) by setting the laser to work in the CW mode. The wavelength was tunable from 730 to $860 \mathrm{~nm}$. The CW at $632.8 \mathrm{~nm}$ was from a collimated helium-neon laser. In the deflection experiment, the CW beams were focused on the sample and the output diffraction beams were recorded by a CCD camera (General Electric: SITE GARD II) in the far field. In the hologram experiment, the $\mathrm{CW}$ beams were shrunk by a 4-f system before being shone onto the metasurface and the holography images were recorded by a cell phone camera on a whitepaper screen located $11.5 \mathrm{~cm}$ after the sample. The efficiency was measured by a power meter.

\section{Acknowledgements}

This work was supported by the Bill \& Melinda Gates Foundation (OPP1119542), the US Army Research Office (W911NF-15-1-0319), DARPA (W911NF-15-1-0319), and National Science Foundation grants (1701163 and 1722169). The fabrication described in this work was done in part at the Cornell NanoScale Facility, an NNCI member supported by NSF grant ECCS1542081. 


\section{Author contributions}

J.Z. and C.G. discussed and defined the project. J.Z. performed the theoretical simulations, experimental fabrication, and measurements. M.E. assisted in developing the theoretical models and performing the data analyses. R.W. designed and simulated the holographic phase mask. S.C.S. and B.L. assisted with the fabrication and measurements. J.Z., M.E., and C.G. wrote the paper and SI. All the authors commented on the paper

\section{Conflict of interest}

The authors declare that they have no conflict of interest.

Supplementary information is available for this paper at https://doi.org/ 10.1038/s41377-019-0164-8.

Received: 26 March 2019 Revised: 22 May 2019 Accepted: 27 May 2019 Published online: 12 June 2019

\section{References}

1. Meinzer, N., Barnes, W. L. \& Hooper, I. R. Plasmonic meta-atoms and metasurfaces. Nat. Photonics 8, 889-898 (2014).

2. Won, R. The rise of plasmonic metasurfaces. Nat. Photonics 11, 462-464 (2017).

3. $\mathrm{Yu}, \mathrm{N}$. F. et al. Light propagation with phase discontinuities: generalized laws of reflection and refraction. Science 334, 333-337 (2011).

4. Ni, X. J. et al. Broadband light bending with plasmonic nanoantennas. Science 335, 427 (2012).

5. Huang, L. L. et al. Dispersionless phase discontinuities for controlling light propagation. Nano Lett. 12, 5750-5755 (2012).

6. Sun, S. L. et al. High-efficiency broadband anomalous reflection by gradient meta-surfaces. Nano Lett. 12, 6223-6229 (2012).

7. Chen, X. et al. Dual-polarity plasmonic metalens for visible light. Nat. Commun. 3, 1198 (2012).

8. $\mathrm{Ni}, \mathrm{X}$. J. et al. Ultra-thin, planar, Babinet-inverted plasmonic metalenses. Light: Sci. Appl. 2, e72 (2013).

9. Aieta, F. et al. Aberration-free ultrathin flat lenses and axicons at telecom wavelengths based on plasmonic metasurfaces. Nano Lett. 12, 4932-4936 (2012).

10. Wen, D. D. et al. Metasurface device with helicity-dependent functionality. Adv. Opt. Mater. 4, 321-327 (2016).

11. $\mathrm{Yu}, \mathrm{N}$. F. et al. A broadband, background-free quarter-wave plate based on plasmonic metasurfaces. Nano Lett. 12, 6328-6333 (2012).

12. Zhao, Y. \& Alù, A. Tailoring the dispersion of plasmonic nanorods to realize broadband optical meta-waveplates. Nano Lett. 13, 1086-1091 (2013).

13. Karimi, E. et al. Generating optical orbital angular momentum at visible wavelengths using a plasmonic metasurface. Light: Sci. Appl. 3, e167 (2014).

14. Bouchard, F. et al. Optical spin-to-orbital angular momentum conversion in ultra-thin metasurfaces with arbitrary topological charges. Appl. Phys. Lett. 105, 101905 (2014)

15. Zeng, J. W. et al. Generating and separating twisted light by gradient-rotation split-ring antenna metasurfaces. Nano Lett. 16, 3101-3108 (2016).

16. Mehmood, M. Q. et al. Visible-frequency metasurface for structuring and spatially multiplexing optical vortices. Adv. Mater. 28, 2533-2539 (2016).

17. Huang, L. L. et al. Three-dimensional optical holography using a plasmonic metasurface. Nat. Commun. 4, 2808 (2013).

18. Ni, X. J. et al. Metasurface holograms for visible light. Nat. Commun. 4, 2807 (2013).

19. Zheng, G. X. et al. Metasurface holograms reaching $80 \%$ efficiency. Nat. Nanotechnol. 10, 308-312 (2015).

20. Huang, L. L. et al. Broadband hybrid holographic multiplexing with geometric metasurfaces. Adv. Mater. 27, 6444-6449 (2015).

21. Stockman, M. I. Nanoplasmonics: past, present, and glimpse into future. Opt. Express 19, 22029-22106 (2011).

22. Maier, S. A. Plasmonics: Fundamentals and Applications (Springer Verlag, Berlin, 2007).

23. Stockman, M. I. et al. Roadmap on plasmonics. J. Opt. 20, 043001 (2018)

24. Kawata, S., Inouye, Y. \& Verma, P. Plasmonics for near-field nano-imaging and superlensing. Nat. Photonics 3, 388-394 (2009).

25. Tame, M. S. et al. Quantum plasmonics. Nat. Phys. 9, 329-340 (2013).

26. Kauranen, M. \& Zayats, A. V. Nonlinear plasmonics. Nat. Photonics 6, 737-748 (2012).
27. Atwater, H. A. \& Polman, A. Plasmonics for improved photovoltaic devices. Nat. Mater. 9, 205-213 (2010).

28. Xie, Z. H. et al. Plasmonic nanolithography: a review. Plasmonics 6, 565 (2011)

29. Stewart, M. E. et al. Nanostructured plasmonic sensors. Chem. Rev. 108 494-521 (2008)

30. Xin, H. B., Namgung, B. \& Lee, L. P. Nanoplasmonic optical antennas for life sciences and medicine. Nat. Rev. Mater. 3, 228-243 (2018).

31. Yu, N. F. \& Capasso, F. Flat optics with designer metasurfaces. Nat. Mater. 13, 139-150 (2014).

32. Chen, W. T. et al. A broadband achromatic metalens for focusing and imaging in the visible. Nat. Nanotechnol. 13, 220-226 (2018).

33. Wang, S. M. et al. A broadband achromatic metalens in the visible. Nat. Nanotechnol. 13, 227-232 (2018)

34. Devlin, R. C. et al. Broadband high-efficiency dielectric metasurfaces for the visible spectrum. Proc Natl Acad. Sci. USA 113, 10473-10478 (2016)

35. Arbabi, A. \& Faraon, A. Fundamental limits of ultrathin metasurfaces. Sci. Rep. 7, 43722 (2017).

36. Monticone, F., Estakhri, N. M. \& Alù, A. Full control of nanoscale optical transmission with a composite metascreen. Phys. Rev. Lett. 110, 203903 (2013).

37. Kruk, S. \& Kivshar, Y. Functional meta-optics and nanophotonics governed by mie resonances. ACS Photonics 4, 2638-2649 (2017).

38. Ding, F., Pros, A. \& Bozhevolnyi, S. I. Gradient metasurfaces: a review of fundamentals and applications. Rep. Prog. Phys. 81, 026401 (2018).

39. Wan, W. W., Gao, J. \& Yang, X. D. Metasurface holograms for holographic imaging. Adv. Opt. Mater. 5, 1700541 (2017).

40. Zhang, $L$. et al. Advances in full control of electromagnetic waves with metasurfaces. Adv. Opt. Mater. 4, 818-833 (2016)

41. He, Q. et al. High-efficiency metasurfaces: principles, realizations, and applications. Adv. Opt. Mater. 6, 1800415 (2018).

42. Epstein, A. \& Eleftheriades, G. V. Huygens' metasurfaces via the equivalence principle: design and applications. J. Opt. Soc. Am. B 33 A31-A50 (2016).

43. Decker, M. et al. High-efficiency dielectric Huygens' surfaces. Adv. Opt. Mater. 3, 813-820 (2015).

44. Kerker, M., Wang, D. S. \& Giles, C. L. Electromagnetic scattering by magnetic spheres. J. Opt. Soc. Am. 73, 765-767 (1983).

45. Pfeiffer, C. \& Grbic, A. Metamaterial Huygens' surfaces: tailoring wave fronts with reflectionless sheets. Phys. Rev. Lett. 110, 197401 (2013).

46. Pfeiffer, C. \& Grbic, A. Millimeter-wave transmitarrays for wavefront and polarization control. IEEE Trans. Microw. Theory Tech. 61, 4407-4417 (2013).

47. Pfeiffer, C. et al. Efficient light bending with isotropic metamaterial Huygens' surfaces. Nano Lett. 14, 2491-2497 (2014).

48. Wong, J. P. S. et al. Design of unit cells and demonstration of methods for synthesizing Huygens metasurfaces. Photonics Nanostruct.Fundam. Appl. 12, 360-375 (2014).

49. Cheng, $\mathrm{H}$. et al. Emergent functionality and controllability in few-layer metasurfaces. Adv. Mater. 27, 5410-5421 (2015).

50. Chen, S. Q. et al. Empowered layer effects and prominent properties in fewlayer metasurfaces. Adv. Opt. Mater. 7, 1801477 (2019).

51. Grady, N. K. et al. Terahertz metamaterials for linear polarization conversion and anomalous refraction. Science 340, 1304-1307 (2013).

52. Zhu, W. M. et al. A flat lens with tunable phase gradient by using random access reconfigurable metamaterial. Adv. Mater. 27, 4739-4743 (2015).

53. El Kabbash, M. et al. Plasmon-exciton resonant energy transfer: across scales hybrid systems. J. Nanomater. 2016, 4819040 (2016).

54. Liu, W. \& Kivshar, Y. S. Generalized Kerker effects in nanophotonics and metaoptics [Invited]. Opt. Express 26, 13085-13105 (2018).

55. Wen, D. D. et al. Metasurface for characterization of the polarization state of light. Opt. Express 23, 10272-10281 (2015).

56. Alu, A. \& Engheta, N. How does zero forward-scattering in magnetodielectric nanoparticles comply with the optical theorem? J. Nanophotonics 4, 041590 (2010).

57. Pors, A. et al. Unidirectional scattering by nanoparticles near substrates: generalized Kerker conditions. Opt. Express 23, 28808-28828 (2015).

58. Alaee, R., Rockstuhl, C. \& Fernandez-Corbaton, I. An electromagnetic multipole expansion beyond the long-wavelength approximation. Opt. Commun. 407, 17-21 (2018).

59. Oulton, R. F. et al. A hybrid plasmonic waveguide for subwavelength confinement and long-range propagation. Nat. Photonics 2, 496-500 (2008). 
60. Qin, F. et al. Hybrid bilayer plasmonic metasurface efficiently manipulates visible light. Sci. Adv. 2, e1501168 (2016).

61. Cui, Z. Nanofabrication: Principles, Capabilities and Limits 2nd edn (Springer, Cham, 2017)

62. Burch, J. \& Di Falco, A. Surface topology specific metasurface holograms. ACS Photonics 5, 1762-1766 (2018).

63. Aieta, F. et al. Out-of-plane reflection and refraction of light by anisotropic optical antenna metasurfaces with phase discontinuities. Nano Lett. 12. 1702-1706 (2012).
64. Gerchberg, R. W. \& Saxton, W. O. A practical algorithm for the determination of phase from image and diffraction plane pictures. Optik 35, 237-246 (1972).

65. Lapedes, D. N. Dictionary of Scientific and Technical Terms. 2nd edn (McGraw Hill, New York, 1978).

66. Kruk, S. et al. Invited article: broadband highly efficient dielectric metadevices for polarization control. APL Photonics 1, 030801 (2016).

67. Zhu, A. Y. et al. Giant intrinsic chiro-optical activity in planar dielectric nanostructures. Light: Sci. Appl. 7, 17158 (2018). 